\title{
Adaptive Exploration-based Whale Optimization for Image Segmentation Based on Variable Parametric Error
}

\author{
M. Jogendra Kumar, G.V.S Raj Kumar, K. Naveen Kumar, Y. Srinivas
}

\begin{abstract}
Image segmentation is the process of splitting an image into numerous segments. Its major purpose is to change or simplify the image, which could be more significant and simpler to examine. However, it does not execute well while segmenting complex images with non-homogeneous parts. In this paper, a hybrid image segmentation model with the aid of Active Contour and Graph cut techniques is proposed. Moreover, it extracts the mutual information from two adopted segmentation schemes, and subsequently, the high-intensity and low-intensity pixels of resultant images are grouped by Fuzzy Entropy Maximization (FEM) method. A modified optimization algorithm termed as Adaptive Exploration based Whale Optimization (AEW) is used for solving the FEM problem. The performance of the proposed Active contour Graph cut Fuzzy Entropy-based Segmentation(AGFES), (AEW-AGFES) is algorithmically analyzed in terms of various performance measures to substantiate its effectiveness.
\end{abstract}

Index Terms: Adaptiveness; Whale Optimization; Image segmentation; Active contour; Graph cut technique; Fuzzy Entropy Maximization Nomenclature

\begin{tabular}{|c|l|}
\hline Acronyms & \multicolumn{1}{|c|}{ Descriptions } \\
\hline FCM & Fuzzy C-Means \\
\hline GM & Gaussian mixture \\
\hline FPR & False Positive Rate \\
\hline FNR & False Negative Rate \\
\hline NPV & Net present Value \\
\hline FDR & False Discovery Rate \\
\hline MCC & Matthew's Correlation Coefficient \\
\hline
\end{tabular}

\section{INTRODUCTION}

Image segmentation [1] [2] [23] [24] remains a challenging issue in computer vision with a variety of appliances. The aim of segmentation is to separate an image into areas of homogeneous features, which refers to the objects parts or objects. On considering the traditional schemes, unsupervised image segmentation [3] [4] has been one of the extensively deployed techniques. The inspiration of this scheme was to split an input image into a compilation of blocks and subsequently, it clusters the blocks in relation

Revised Manuscript Received on April 12, 2019.

M. Jogendra Kumar, Department of Computer Science and Engineering, Gandhi Institute of Technology and Management, GITAM (Deemed to be University), Vishakhapatnam, A.P, India

Dr. G.V.S Raj Kumar, Department of Information Technology, Gandhi Institute of Technology and Management, GITAM (Deemed to be University), Vishakhapatnam, A.P, India

Dr. K.Naveen Kumar, Department of Information Technology, Gandhi Institute of Technology and Management, GITAM (Deemed to be University), Vishakhapatnam, A.P, India

Dr.Y.Srinivas, Department of Information Technology, Gandhi Institute of Technology and Management, GITAM (Deemed to be University), Vishakhapatnam, A.P India with certain image features for attaining segmentation outcomes [5] [6] [7]. Conventional characteristics namely, co-occurrence, energy, moment were established and extensively implemented in diverse fields, however, the outcomes are not much remarkable in segmentation appliances [8] [9].

The experimentation exposed that the distribution of image deviations characterizes the contents of the image and shows potential outcomes in classification and retrieval [10] [11]. On the other hand, the distributions are generally high-dimensional that formulates them to less proficient in real time appliances. Therefore discovering an effectual arithmetical model for demonstrating such image deviations is significant and essential. From its initiation, FEM has attained great consideration from experts on these techniques [12] [13]. FEM the renowned clustering technique [14] [15], and its improved models were executed as authoritative tools due to its fast convergence, performance and simplicity [16] [17]. In the previous decades, several reseraches have been undertaken on image segmentation using different techniques. Generally, FEM could attain excellent segmentation accuracy while dealing low or no noise. However, the segmentation [18] [19] outcomes worsen rapidly with the increase in image noise level. For minimizing the worse impacts of noise in fuzzy approach, the better solution is to carry out "image denoising" on the smoothened image.

This paper contributes a hybrid image segmentation approach using Active Contour and Graph cut techniques. In this paper, the high-intensity and low-intensity pixels of the mutual informative image are grouped by FEM scheme. Here, a modified optimization technique named as AEW is deployed for resolving the optimization problem in FEM. The performance of the adopted AEW-AGFES is further algorithmically examined with respect to different performance measures for validating its efficiency. The paper is organized as follows. Section II portrays the literature work. Section III explains the adopted image segmentation procedure, and Section IV explains the adopted contribution of image segmentation. Section V discusses the experimental results, and Section VII concludes the paper. 


\section{ADAPTIVE EXPLORATION-BASED WHALE OPTIMIZATION FOR IMAGE SEGMENTATION BASED ON VARIABLE PARAMETRIC ERROR}

\section{LITERATURE REVIEW}

\section{Related works}

In 2019, Choy et al. [1] have introduced a new fuzzy-based image segmentation technique. Here, probability model was established for portraying the variations of image distributions on the basis of bit-plane dependencies and probabilities among the bit-planes. The conventional image segmentation models demonstrates that the distributions include specific structures (e.g., symmetry, monotone and periodicity), whereas the adopted technique offers a widespread parametric demonstration, which could be deployed for modeling random distributions without implementing any particular limitations regarding the distributions. Finally, the experimentations exhibit the advanced performance of the introduced technique.

In 2018, Guo et al. [2] have modeled a wide-ranging scheme to develop the noisy image segmentation using fuzzy clustering approach by incorporating the guided filter in a novel manner. In particular, the fuzzy clustering was deployed on the smoothened image for attaining more standardized segments; however, the real noisy image was deployed for directing the guided filters for post-processing the memberships of fuzzy approach. On performing this, the loss of information could be reduced. Experimentation on real and synthetic images reveals that the introduced framework could overcome the conventional fuzzy clustering schemes considerably with reduced run-time overhead.

In 2016, Peng Gu et al. [3] have adopted an automated approach for the segmentation of all 3D ultrasound volumes. The segmentation was done into three types namely 'mass/cist' tissue, 'fatty tissue', and 'fibro-glandular tissue'. In addition, they have investigated the efficiency as well as the consistency of proposed model. Results of the experiment have reviewed better performance of proposed model by distinguishing fat or non-fat tissues. Altogether, the model has attained perfect consistency with an accuracy of $85.7 \%$.

In 2017, Ji et al. [4], has established a robust modified GM design for segmenting the images. Initially, to formulate the GM schemes, a novel spatial weight factor was set up in its immediate neighborhood. Subsequently, for diminishing the over-smoothness, an approach of prior factor was implemented by integrating the spatial data between neighborhood pixels. At last, all Gaussian components were categorized by three routinely determined rough portions. Finally, the established algorithm was compared with other schemes to reveal the betterment of the established model.

In 2016, Andrea et al. [5] have proposed an approach known as Delaunay Triangulation to take out "binary mask" devoid of the requirement of training phase. In addition, an investigational assessment has been carried out by considering six renowned conventional segmentation techniques. The consequences of the investigational scrutiny had revealed that the suggested approach was extremely precise when managing with benign lesions.

\section{PROPOSED IMAGE SEGMENTATION PROCEDURE}

An active contour [20] is an approach for recognizing the specific features contained in an image. The mathematical form of active contour is specified by Eq. (1), in which $S$ points out the control for normalized index points, and $x(V)$ and $y(V)$ denotes $x, y$ coordinates of contour.

$$
\bar{S}(V)=(\vec{x}(V), \vec{y}(V))
$$

The internal energy corresponds to the elastic energy and bending energy is denoted as specified in Eq. (2), in which $\alpha$ indicates an adjustable constant that indicates continuity and $\beta$ indicates an adjustable constant that denotes curving of contours. The elastic and bending energies are formulated in Eq. (3) and Eq. (4), respectively.

$$
\begin{aligned}
& e_{\text {int }}=e_{\text {elastic }}+e_{\text {bend }}=\alpha(V)\left|\frac{d S}{d V}\right|^{2}+\beta(V)\left|\frac{d^{2} S}{d V^{2}}\right|^{2} \\
& e_{\text {bend }}=\int_{V} \beta(\vec{S}(V-1)-\vec{S}(V)+\vec{S}(V+1))^{2} d V \\
& e_{\text {elastic }}=\int_{S} \alpha(\vec{S}(V)-\vec{S}(V-1))^{2} d V
\end{aligned}
$$

The reduced energy function is specified in Eq. (5), in which internal energy, picture's energy and the external limitations of curve is given by $e_{\mathrm{int}}, e_{\text {image }}$ and $e_{\text {con }}$. $e_{\text {snake }}^{*}=\int_{0}^{1} e_{\text {snake }}(S(V)) d V=\int_{0}^{1}\left\{e_{\text {int }}(S(V))+e_{\text {image }}(S(V))+e_{\text {con }}(S(V))\right\} d V$

Thus the active contour-based segmented image is indicated by $B(x, y)$.

\section{Graph cut Segmentation:}

Graph cuts [21] is deployed for resolving several low-level computer vision problems namely, image segmentation, image smoothing, the stereo correspondence crisis and various issues that are computed concerning the minimization of energy. Assume $G=\left\{g_{1}, g_{2}, g_{3} \ldots . g_{p}\right\}$ in which $p$ denotes the count of image pixels and $g_{i} \in\{0,1\}$. The energy function is minimized by the min-cut as given in Eq. (6), in which, $r(G)$ is said to be the regional parameter and $b(G)$ is indicated as the boundary parameter and the equivalent constraint among regional term and boundary is termed as $\delta$.

$$
L(G)=\delta r(G)+b(G)
$$

The energy function in regional constraint is denoted by Eq. (7), in which, $r_{p}\left(g_{p}\right)$ denotes the consequence for allocating label $g_{p}$ to pixel ${ }^{p}$. Eq. (8) and Eq. (9) shows the weight of $t$-links.

$$
\begin{aligned}
& r(G)=\sum_{p \in P} r_{p}\left(g_{p}\right) \\
& r_{p}(1)=-\ln \operatorname{Pr}\left(I_{p} \mid \text { 'object' }\right)
\end{aligned}
$$




$$
r_{p}(0)=-\ln \operatorname{Pr}\left(I_{p} \mid \text { background }\right)
$$

From Eq. (8) and (9), it is noticed that when

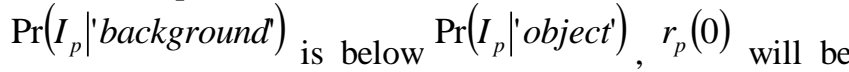
greater than $r_{p}(1)$. In Eq. (10), $b(G)$ denote border term as revealed in Eq. (6), in which $p, q$ denotes neighboring pixels.

$$
\begin{aligned}
& b(G)=\sum_{\{p, q\} \in N} b_{<p, q>} . \delta\left(g_{p}, g_{q}\right) \\
& \text { where, }
\end{aligned}
$$

Accordingly, $\quad b_{<p, q>}$ is regarded as a non increasing function of $g_{p}-g_{q}$ as given by Eq. (11), in which $\sigma$ indicates camera noise. The weight of $s-t$ graph is specified in Eq. (12).

$$
\begin{aligned}
& b_{<p, q>} \alpha \exp \left(\frac{-\left(g_{p}-g_{q}\right)^{2}}{2 \sigma^{2}}\right) \\
& \text { Weight }= \begin{cases}b_{<p, q>} & \{p, q\} \in \text { Neighbouring pixel } \\
\alpha \cdot r_{p}(0) & \text { for edge }(p, s) \\
\alpha \cdot r_{p}(1) & \text { for edge }(p, t)\end{cases}
\end{aligned}
$$

Thus the two segmented output from graph cut algorithm is indicated by $C(x, y)$ and $D(x, y)$.

\section{Mutual segmentation:}

After partitioning the image, $I(x, y)$ using Graph cut method, the resulting Graph cut images, $C(x, y)$ and $D(x, y)$ is correlated with active contour image $B(x, y)$. The common information of images, $C(x, y)$ and $B(x, y)$ is attained, and is indicated by $E(x, y)$. Similarly, mutual information of $D(x, y)$ and $B(x, y)$ are attained and is denoted as $A(x, y)$.

\section{Grouping low and high-intensity pixels:}

Here, the low and high intensity of the images $E(x, y)$ and $A(x, y)$ are split using FEM model. The fuzzy 2-partition entropy is given by Eq. (13).

$$
F=-H_{d} \log \left(H_{d}\right)-H_{b} \log \left(H_{b}\right)
$$

The fuzzy entropy is updated as given in Eq. (13), since the bright set $H_{b}$ can be taken as $H_{b}=1-H_{d}$. The value of $H_{d}$ is updated as revealed in Eq. (14), in which $u, v, w$ denotes the shape of $W$ membership functions. The value of $F$ in Eq. (15) denotes both $F_{1}$ and $F_{2}$, where $F_{1}=$ $F(E(x, y))$ and $F_{2}=F(A(x, y))$.

$$
F=-H_{d} \log \left(H_{d}\right)-\left(1-H_{d}\right) \log \left(1-H_{d}\right)
$$

$$
\begin{aligned}
& H_{d}=\frac{1}{(w-u)(w-v)} \sum_{k=v+1}^{w}(k-w)^{2} h(k)- \\
& \frac{1}{(w-u)(v-u)} \sum_{k=u+1}^{v}(k-u)^{2} h(k)+\sum_{k=0}^{v} h(k)
\end{aligned}
$$

The optimal set of constraints that increases the $F$ value is chosen. Thus the images $E(x, y)$ and $A(x, y)$ are split into low-intensity images $E_{1}(x, y)$ and $A_{1}(x, y)$ and high-intensity images of $E_{2}(x, y)$ and $A_{2}(x, y)$ respectively.

\section{ADOPTED CONTRIBUTION OF IMAGE SEGMENTATION}

\section{Proposed Architecture}

As per the proposed method, an image $I(x, y)$ is subjected to active contour model, where a mask is deployed for segmenting the given image. By exploiting the mask, the optimal region could be segmented separately that is denoted by $B(x, y)$. In addition, the original image $I(x, y)$ is given to graph cut scheme that split the image into two segments. $C(x, y)$ and $D(x, y)$. The two divisions are further compared with active contour image $B(x, y)$, and the mutual information of two images with $B(x, y)$ is taken, which is denoted as $E(x, y)$ and $A(x, y)$. Moreover, FEM helps to separate the low intensity, and high intensity pixels of both $E(x, y)$ and $A(x, y)$ images. Accordingly, the low intensity images are indicated as $E_{1}(x, y)$ and $A_{1}(x, y)$, and high intesnity images are indicated as $E_{2}(x, y)$ and $A_{2}(x, y)$. As the main contribution, the maximization problem in FEM is resolved by AEW scheme for obtaining the high and low intensity pixels. Here, AEW optimizes $u, v$ and $w$ of the membership function of fuzzy 2-partition entropy. The best segmented part can be attained from any of the four images. The overall framework of the adopted image segmentation model is given by Fig. 1. 


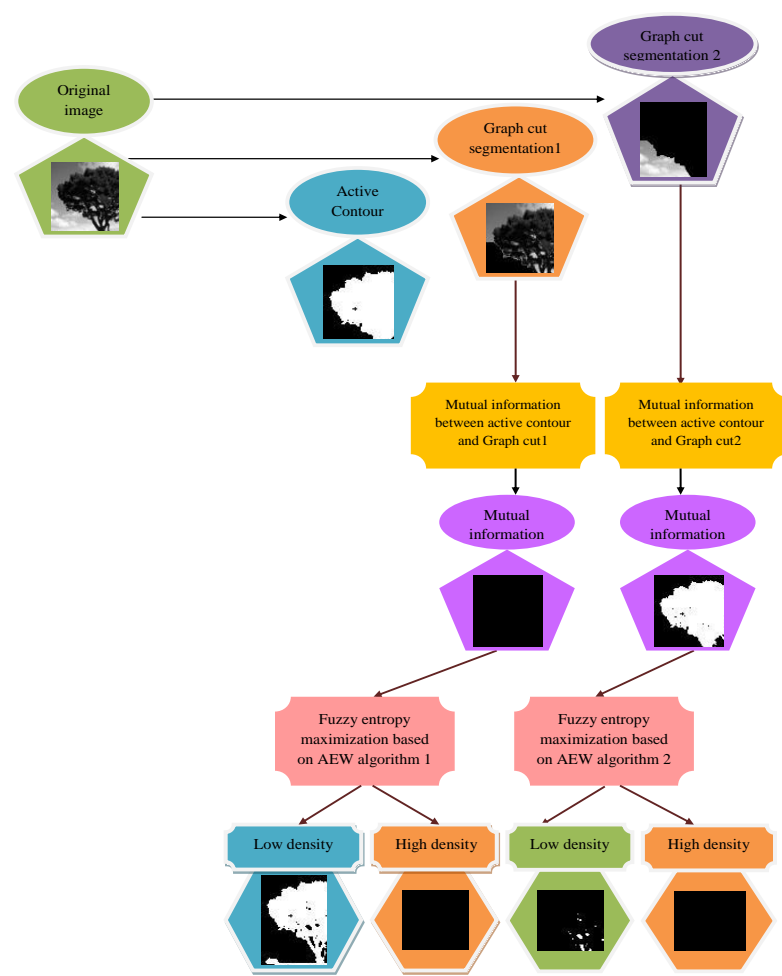

Fig. 1 Architectural representation of the proposed image segmentation model

The fuzzy 2-partition entropy function of the adopted AEW-AGFES method is denoted by $F$. The variables $u, v, w$, together indicated by $X$ that denotes the shape of $W$ membership function of fuzzy is provided as solution to AEW scheme as shown by Fig. 2, where the objective is to maximize the entropy $F$ as specified in Eq. (16).

Objective $=\operatorname{Max}(F)$

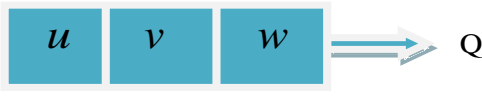

Fig.2 Solution Encoding

\section{Proposed AEW model}

The fuzzy entropy constraints $u, v, w$ are offered to AEW model for optimization. In general, whales [22] have the ability to identify the location of prey and encircle them. This feature is mathematicaly indicated by Eq. (17) and Eq. (18), where, $t$ refers to the present iteration, the position vector of whale is indicated by $Q$, position vector of prey is indicated by $Q^{*}, \vec{U}$ and $\vec{Q}$ denotes the vector coefficient, '.' denotes element-by-element multiplication and || depicts the absolute value.

$$
\begin{aligned}
& \vec{E}=\left|\vec{U} \cdot \vec{Q}^{*}(t)-\vec{Q}(t)\right| \\
& \vec{Q}(t+1)=\vec{Q}^{*}(t)-\vec{X} \cdot \vec{E}
\end{aligned}
$$

It is essential to observe that $Q^{*}$ has to be updated in the entire iterations with an improved solution. The vectors $\vec{X}$ and $\vec{U}$ are estimated as given by Eq. (19) and Eq. (20) in which the value of $\vec{a}$ is linearly minimized from 2 to 0 for further iterations and $\vec{v}$ indicates a random vector, which lies among 0 and 1 .

$$
\begin{aligned}
\vec{X} & =2 \vec{a} \cdot \vec{v}-\vec{a} \\
\vec{U} & =2 \cdot \vec{v}
\end{aligned}
$$

\section{Exploitation Phase:}

Shrinking encircling method: This phase is carried out by minimizing the value of $\vec{a}$ in Eq. (18). In addition, note that the variation of $\vec{X}$ is minimized by $\vec{a}$, here $\vec{X}$ lies between $[-\vec{a}, \vec{a}]$ in a random manner and $\vec{a}$ is reduced from 2 to 0 for more iterations.

Spiral updating location: The distance between the whales positioned at $(Q, Y)$ and prey positioned at $\left(Q^{*}, Y^{*}\right)$ are estimated in this process. A spiral model is included for identifying the location of prey and whale as given in Eq. (21) in which $\vec{E}^{\prime}=\left|\vec{Q}^{*}(t)-\vec{Q}(t)\right|$ and it specifies the distance of $i^{\text {th }}$ whale's towards the prey, the constraint for relating the logarithmic spiral shape is shown by $u$, and $h$ refers to an varying constraint that lies among $[-1,1]$ and $\tau=0.5$.

$$
\begin{aligned}
& \vec{Q}(t+1)=\vec{E} \cdot e^{u h} \cdot \cos (2 \pi h)+\vec{Q}^{*}(t) \\
& \vec{Q}(t+1)=\left\{\begin{array}{l}
\vec{Q}^{*}(t)-\vec{X} \cdot \vec{E} \quad \text { if } p e<\tau \\
\vec{E}^{\prime} \cdot e^{u h} \cdot \cos (2 \pi h)+\vec{Q}^{*}(t) \quad \text { if } p e \geq \tau
\end{array}\right.
\end{aligned}
$$

The final updated formula is based on Eq. (22) where $p e$ denotes parametric error that lies among 0 and 1 .

\section{Exploration Phase:}

Here, a randomly chosen searching agent is recognized instead of the most excellent search agent. Here, $|\vec{X}|>1$ emphasizes the exploration process and permits the adopted system to perform a global search as given by Eq. (23) and Eq. (24), where $\vec{Q}_{\text {rand }}$ denotes a arbitrary whale, that is selected from the population existed.

$$
\begin{gathered}
\vec{E}=\left|\vec{U} \cdot \vec{Q}_{\text {rand }}-\vec{Q}\right| \\
\vec{Q}(t+1)=\vec{Q}_{\text {rand }}-\vec{X} \cdot \vec{E}
\end{gathered}
$$

The proposed AEW algorithm is enhanced by establishing a bounding factor, denoted by $O$ as presented in Eq. (25), in which $l b$ refers to the lower bound and $u b$ denotes the upper bound, and the maximum iteration is referred by $\operatorname{Max}(t)$ Here, the distance indicated by $D$ between the best position and current position is specified by Eq. (26).

$$
\begin{aligned}
& o=(u b-l b) / 4+(u b-l b) \times[(t+1 / \operatorname{Max}(t)) \times 2] \\
& D=\mid \text { Current position-Best position } \mid
\end{aligned}
$$


solution constraints in $D$ compared with $O$ is checked and is indicated by $r_{s} \quad$ Depending on $r_{s}$, the vectors $\vec{X}$ and $\vec{U}$ are approximated as shown in Eq. (27) and Eq.(28) by means of $\vec{v}_{1}$ and $\vec{v}_{2}$ denoted in Eq. (29) and Eq. (30), correspondingly.

$$
\begin{aligned}
\vec{X} & =2 \vec{a} \cdot \vec{v}_{1}-\vec{a} \\
\vec{U} & =2 \cdot \vec{v}_{2} \\
\vec{v}_{1} & =\left[\operatorname{length}(o) / \operatorname{length}\left(r_{s}\right)+0.01\right]+\operatorname{rand}_{1}() \\
\vec{v}_{2} & =\left[\operatorname{length}(o) / \operatorname{length}\left(r_{s}\right)+0.01\right]+\operatorname{rand}_{2}()
\end{aligned}
$$

\begin{tabular}{|c|c|}
\hline & Algorithm 1: AEW-AGFES algorithm \\
\hline Step1 & Assign whale's population $Q_{i}(i=1,2, \ldots ., n)$ \\
\hline Step2 & Find out fitness values of all exploring agents \\
\hline Step3 & The most desired agent for search is $Q^{*}$ \\
\hline Step4 & $\begin{array}{l}\text { While } t \text { is lower than total iterations } \\
\text { For entire searching agents }\end{array}$ \\
\hline Step5 & Determine $O$ as per Eq. (25) \\
\hline Step6 & Compute distance $D$ as per Eq. (26) \\
\hline Step7 & Find $\vec{v}_{1}$ and $\vec{v}_{2}$ as per Eq. (29) and Eq. (30). \\
\hline Step8 & Update $a, Q, U, h$ and $p e$ \\
\hline Step9 & if $1 p e<\tau$ \\
\hline Step10 & if $2(|X|<1)$ \\
\hline Step11 & $\begin{array}{l}\text { Position of current search agent is } \\
\text { updated as per Eq. (18) } \\
\text { else if } 2(|X| \geq 1)\end{array}$ \\
\hline & $\begin{array}{l}\text { Choose a random search agent } \vec{Q}_{\text {rand }} \\
\text { Update position of present search } \\
\text { agent as per Eq. (24) } \\
\text { end if } 2\end{array}$ \\
\hline Step12 & $\begin{array}{l}\text { else if } 1 p e \geq \tau \\
\text { Update position of present search } \\
\text { agent as per Eq. (21) }\end{array}$ \\
\hline & $\begin{array}{l}\text { end if } 1 \\
\text { end for }\end{array}$ \\
\hline Step13 & $\begin{array}{l}\text { Verify if any search agent exceeds the search } \\
\text { space }\end{array}$ \\
\hline Step14 & Evaluate fitness value of search agent \\
\hline Step15 & Update $Q^{*}$ if there is an enhanced solution \\
\hline Step16 & $t=t+1$ \\
\hline & end while \\
\hline & return $Q^{*}$ \\
\hline
\end{tabular}

The below Algorithm 1 depicts the pseudo code for the adopted AEW scheme.

\section{RESULTS AND DISCUSSIONS}

\section{Experimental Set up}

The proposed AEW-AGFES-based image segmentation model was implemented in MATLAB. The database Weizmann was used that was downloaded from "http://www.wisdom.weizmann.ac.il/ vision/Seg_Evaluatio
n_DB/". It comprises of seven sets of images that consists animals, birds, nature, objects, transportation, and tree. In every set, few images were taken for this experiment, i.e., 12 images were considered for birds, 15 images were considered for animals, 26 images were considered for objects, 9 images were considered for transportation and 10 images were considered for tree. Here, algorithmic analysis was held by varying the values of from $=0.25,=0.40,=0.55,=0.70$ and $=0.85$ and performance measures like accuracy, sensitivity,

\begin{tabular}{|c|c|c|c|}
\hline $\begin{array}{c}\text { Image } \\
\text { types }\end{array}$ & Original & $\begin{array}{c}\text { AEW-AGF } \\
\text { ES }\end{array}$ & $\begin{array}{c}\text { Proposed } \\
\text { segmented } \\
\text { images }\end{array}$ \\
\hline Animal & & & \\
\hline Bird & & & \\
\hline $\begin{array}{l}\text { Buildin } \\
\mathrm{g}\end{array}$ & & & \\
\hline Nature & & & \\
\hline Object & & & \\
\hline $\begin{array}{l}\text { Transp } \\
\text { ortation }\end{array}$ & & & \\
\hline Tree & & & \\
\hline & (a) & (b) & c) \\
\hline
\end{tabular}
specificity, and precision, FPR, FNR, NPV, FDR, F1-score, and MCC are analyzed. The segmentation output of adopted method is revealed in Fig. 3.

Fig.3 Sample images for each dataset and its corresponding segmented output for (a) Original image (b) AEW-AGFES-based segmented image (c) proposed segmented images 


\section{ADAPTIVE EXPLORATION-BASED WHALE OPTIMIZATION FOR IMAGE SEGMENTATION BASED ON VARIABLE PARAMETRIC ERROR}

\section{Algorithmic Analysis \& RESULTS}

The algorithmic analysis of the suggested AEW-AGFES method for image segmentation is specified in Fig. 4. In Fig. $4(\mathrm{a})$, the accuracy of adopted model at $6^{\text {th }}$ test case for $\tau=0.25$ is $2.5 \%$ superior to $\tau=0.40,2.5 \%$ superior to $\tau=0.55,2.5 \%$ superior to $\tau=0.70$ and $2.5 \%$ superior to $\tau=0.85$.

From Fig. 4(b), the sensitivity of the implemented scheme at $1^{\text {st }}$ test case for $\tau=0.40$ is $2.17 \%$ superior to $\tau=0.25$, $2.17 \%$ superior to $\tau=0.55,4.44 \%$ superior to $\tau=0.70$ and $2.17 \%$ superior to $\tau=0.85$. Also, at $5^{\text {th }}$ test case, the implemented scheme for $\tau=0.85$ is $2.86 \%$ superior to $\tau=0.25,2.86 \%$ superior to $\tau=0.55$ and $2.86 \%$ superior to $\tau=0.70$. In addition, at $8^{\text {th }}$ test case, the presented system for $\tau=0.85$ is $2.7 \%$ superior to $\tau=0.25,2.7 \%$ superior to $\tau=0.55$ and $2.7 \%$ superior to $\tau=0.70$.

From Fig. 4(c), the specificity of the implemented process at $4^{\text {th }}$ test case for $\tau=0.25$ is $1.19 \%$ superior to $\tau=0.40$, $1.19 \%$ better than $\tau=0.55,1.19 \%$ better than $\tau=0.70$ and $1.19 \%$ better than $\tau=0.85$. Moreover, at $5^{\text {th }}$ test case, the implemented scheme for $\tau=0.85$ is $1.19 \%$ better than $\tau=0.25,1.19 \%$ better than $\tau=0.55,1.19 \%$ better than $\tau=0.40$ and $1.19 \%$ better than $\tau=0.70$. In addition, at $6^{\text {th }}$ test case, the presented system for $\tau=0.85$ is $1.19 \%$ better than $\tau=0.25, \tau=0.55, \tau=0.40$ and $\tau=0.70$. Also at $7^{\text {th }}$ test case, the presented system for $\tau=0.85$ is $1.19 \%$ superior to $\tau=0.25, \tau=0.55, \tau=0.40$ and $\tau=0.70$.

From Fig. 4(d), at $1^{\text {st }}$ test case, the implemented scheme for $\tau=0.85$ is $1.29 \%$ better than $\tau=0.25,1.29 \%$ better than $\tau=0.55,1.29 \%$ better than $\tau=0.40$ and $1.29 \%$ better than $\tau=0.70$. In addition, on considering $7^{\text {th }}$ test case, the implemented scheme for $\tau=0.85$ is $2.78 \%$ better than $\tau=0.25,2.78 \%$ better than $\tau=0.55,2.78 \%$ better than $\tau=0.40$ and $2.78 \%$ better than $\tau=0.70$. Also, FPR of the suggested model can be attained from

Fig. 4(e), where the adopted model at $1^{\text {st }}$ test case, for $\tau=0.70$ is $9.61 \%$ superior to $\tau=0.25,9.61 \%$ superior to $\tau=0.55,9.61 \%$ superior to $\tau=0.40$ and $9.61 \%$ superior to $\tau=0.70$. Moreover, at $2^{\text {nd }}$ test case, the proposed scheme for $\tau=0.70$ is $3.23 \%$ superior to $\tau=0.25,3.23 \%$ superior to $\tau=0.55,3.23 \%$ superior to $\tau=0.40$ and $3.23 \%$ superior to $\tau=0.70$.

Accordingly, from Fig. 4(f), the adopted scheme on considering $7^{\text {th }}$ test case, for $\tau=0.85$ is $1.54 \%$ better than $\tau=0.25,1.54 \%$ better than $\tau=0.55,1.54 \%$ better than $\tau=0.40$ and $1.54 \%$ better than $\tau=0.70$.

From Fig. $4(\mathrm{~g})$, the NPV of the adopted scheme at $5^{\text {th }}$ test case and $6^{\text {th }}$ test case for $\tau=0.85$ is $1.09 \%$ superior to $\tau=0.25,1.09 \%$ superior to $\tau=0.55,1.09 \%$ superior to $\tau=0.40$ and $1.09 \%$ superior to $\tau=0.70$.

In addition, the FDR, F1-score and MCC of the adopted model for varying values of $\tau$ from $\tau=0.25, \tau=0.40, \tau$ $=0.55, \tau=0.70$ and $\tau=0.85$ are portrayed by Fig. 4(h), Fig. 4(i) and Fig. 4(j).

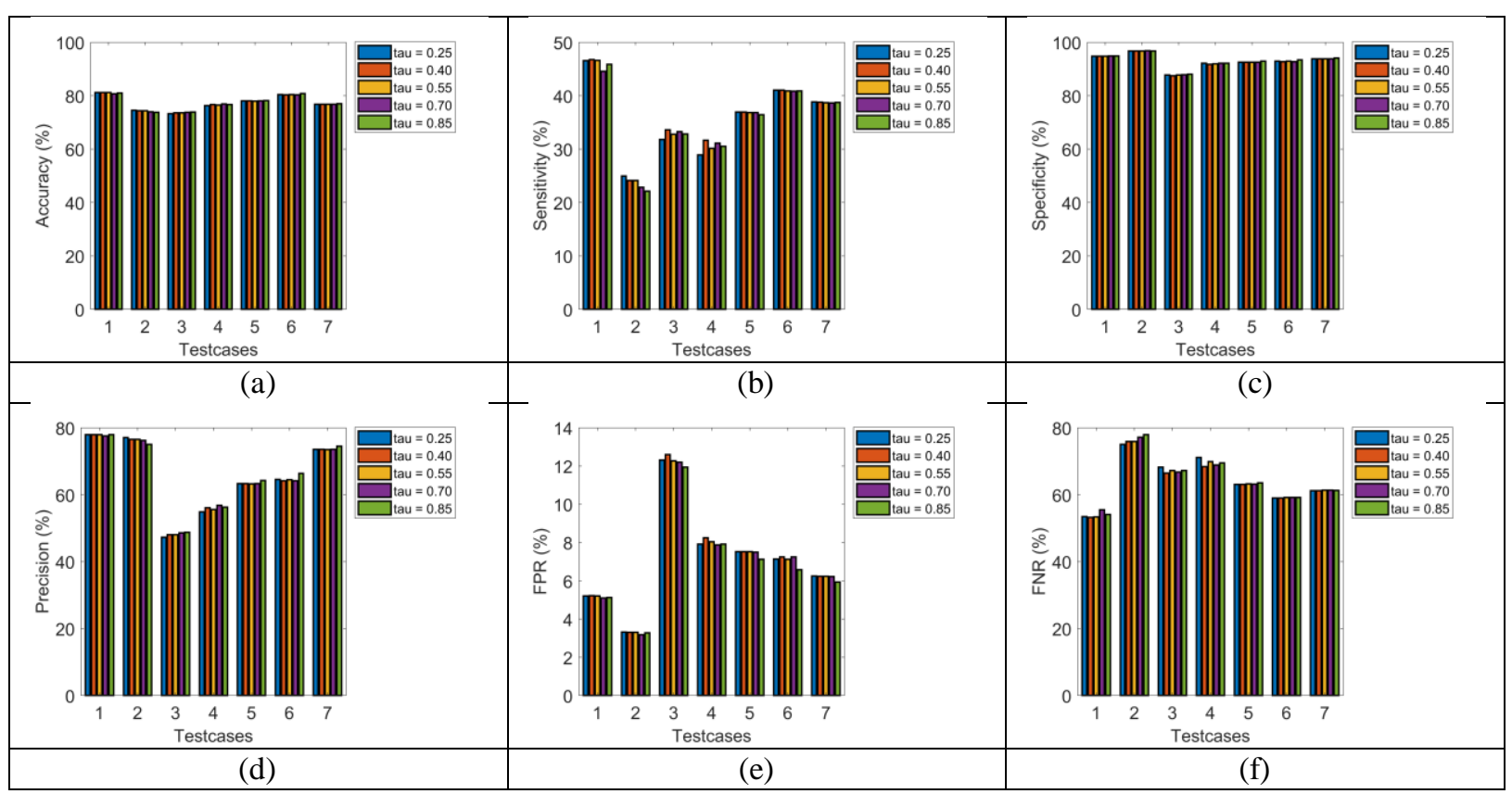




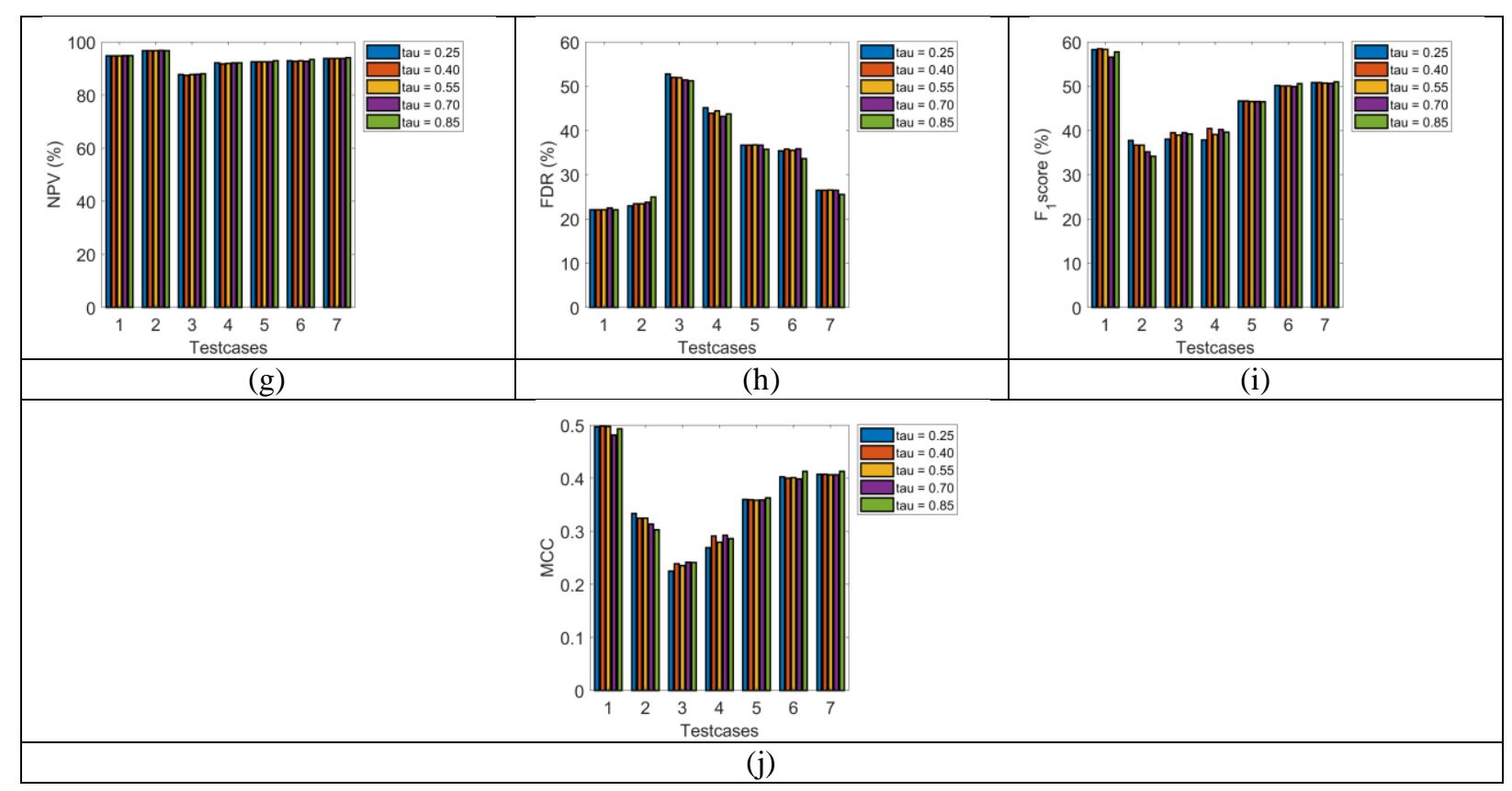

Fig. 4. Performance analysis of the proposed model in terms of (a) Accuracy (b) Sensitivity (c) Specificity (d) Precision (e) FPR (f) FNR (g) NPV (h) FDR (i) F1 core (j) MCC

\section{Overall Performance Analysis}

The performance analysis of the implemented image segmentation scheme when evaluated by varying the values of $\tau$ from $\tau=0.25, \tau=0.40, \tau=0.55, \tau=0.70$ and $\tau=0.85$ for seven test cases is specified from Table I to Table VII, respectively.

Accordingly, from Table I, for test case 1, the accuracy of the proposed scheme for $\tau=0.40$ is $5.91 \%$ better than $\tau=0.25,3.69 \%$ better than $\tau=0.55,0.66 \%$ better than $\tau=0.70$ and $0.21 \%$ better than $\tau=0.85$. In the same way, the sensitivity of the implemented scheme for $\tau=0.40$ is $0.47 \%$ superior to $\tau=0.25,0.36 \%$ superior to $\tau=0.55$, $4.77 \%$ superior to $\tau=0.70$ and $1.99 \%$ superior to $\tau=0.85$.

From Table II, for test case 2, the specificity of the presented design for $\tau=0.70$ is $0.14 \%$ better than $\tau=0.25$, $0.12 \%$ better than $\tau=0.40,0.12 \%$ better than $\tau=0.55$ and
$0.92 \%$ better than $\tau=0.85$. Also, the precision of the adopted scheme for $\tau=0.25$ is $0.65 \%$ superior to $\tau=0.40$, $0.64 \%$ superior to $\tau=0.55,1.09 \%$ superior to $\tau=0.70$ and $2.62 \%$ superior to $\tau=0.85$.

From Table III, for test case 3, the FPR of the proposed scheme for $\tau=0.85$ is $2.93 \%$ better than $\tau=0.25,5.16 \%$ better than $\tau=0.40,2.68 \%$ better than $\tau=0.55$ and $2.14 \%$ better than $\tau=0.70$. In addition, for test case 3, the FPR of the proposed scheme for $\tau=0.40$ is $2.62 \%$ superior to $\tau=0.25,1.22 \%$ superior to $\tau=0.55,0.5 \%$ superior to $\tau=0.70$ and $1.16 \%$ superior to $\tau=0.85$. Similarly, the same analysis is repeated for all test cases, and the proposed performance efficacy has been proven.

TABLE 1. ALGORITHMIC ANALYSIS ON TEST CASE 1

\begin{tabular}{|l|c|c|c|c|c|}
\hline Measures & $\tau=0.25$ & $\tau=0.40$ & $\tau=0.55$ & $\tau=0.70$ & $\tau=0.85$ \\
\hline Accuracy & 0.81145 & 0.81193 & 0.81163 & 0.80657 & 0.81021 \\
\hline Sensitivity & 0.46536 & 0.46757 & 0.46589 & 0.44527 & 0.45891 \\
\hline Specificity & 0.94792 & 0.94772 & 0.94796 & 0.94904 & 0.94874 \\
\hline Precision & 0.77894 & 0.7791 & 0.77925 & 0.77504 & 0.77925 \\
\hline FPR & 0.052078 & 0.052276 & 0.052043 & 0.050963 & 0.051265 \\
\hline FNR & 0.53464 & 0.53243 & 0.53411 & 0.55473 & 0.54109 \\
\hline NPV & 0.94792 & 0.94772 & 0.94796 & 0.94904 & 0.94874 \\
\hline FDR & 0.22106 & 0.2209 & 0.22075 & 0.22496 & 0.22075 \\
\hline F1-score & 0.58264 & 0.58441 & 0.58314 & 0.56559 & 0.57764 \\
\hline MCC & 0.49672 & 0.49824 & 0.49725 & 0.4814 & 0.49276 \\
\hline
\end{tabular}



VARIABLE PARAMETRIC ERROR

TABLE 2. ALGORITHMIC ANALYSIS ON TEST CASE 2

\begin{tabular}{|l|c|c|c|c|c|}
\hline Measures & $\tau=0.25$ & $\tau=0.40$ & $\tau=0.55$ & $\tau=0.70$ & $\tau=0.85$ \\
\hline Accuracy & 0.74557 & 0.74311 & 0.74311 & 0.73997 & 0.73705 \\
\hline Sensitivity & 0.24975 & 0.24124 & 0.24124 & 0.22846 & 0.22109 \\
\hline Specificity & 0.96679 & 0.96702 & 0.96702 & 0.96817 & 0.96724 \\
\hline Precision & 0.77037 & 0.76543 & 0.76543 & 0.76205 & 0.75071 \\
\hline FPR & 0.033213 & 0.032984 & 0.032984 & 0.031827 & 0.032755 \\
\hline FNR & 0.75025 & 0.75876 & 0.75876 & 0.77154 & 0.77891 \\
\hline NPV & 0.96679 & 0.96702 & 0.96702 & 0.96817 & 0.96724 \\
\hline FDR & 0.22963 & 0.23457 & 0.23457 & 0.23795 & 0.24929 \\
\hline F1-score & 0.37721 & 0.36686 & 0.36686 & 0.35154 & 0.34158 \\
\hline MCC & 0.33335 & 0.32466 & 0.32466 & 0.31349 & 0.30266 \\
\hline
\end{tabular}

TABLE 3. ALGORITHMIC ANALYSIS ON TEST CASE 3

\begin{tabular}{|l|c|c|c|c|c|}
\hline Measures & $\tau=0.25$ & $\tau=0.40$ & $\tau=0.55$ & $\tau=0.70$ & $\tau=0.85$ \\
\hline Accuracy & 0.7331 & 0.73555 & 0.73582 & 0.73758 & 0.73837 \\
\hline Sensitivity & 0.31799 & 0.33584 & 0.32767 & 0.33248 & 0.32802 \\
\hline Specificity & 0.87697 & 0.87408 & 0.87728 & 0.87797 & 0.88058 \\
\hline Precision & 0.47251 & 0.48035 & 0.48062 & 0.48568 & 0.4877 \\
\hline FPR & 0.12303 & 0.12592 & 0.12272 & 0.12203 & 0.11942 \\
\hline FNR & 0.68201 & 0.66416 & 0.67233 & 0.66752 & 0.67198 \\
\hline NPV & 0.87697 & 0.87408 & 0.87728 & 0.87797 & 0.88058 \\
\hline FDR & 0.52749 & 0.51965 & 0.51938 & 0.51432 & 0.5123 \\
\hline F1-score & 0.38015 & 0.3953 & 0.38967 & 0.39473 & 0.39223 \\
\hline MCC & 0.22523 & 0.23891 & 0.23556 & 0.2415 & 0.24105 \\
\hline
\end{tabular}

TABLE 4. ALGORITHMIC ANALYSIS ON TEST CASE 4

\begin{tabular}{|l|c|c|c|c|c|}
\hline Measures & $\tau=0.25$ & $\tau=0.40$ & $\tau=0.55$ & $\tau=0.70$ & $\tau=0.85$ \\
\hline Accuracy & 0.76291 & 0.76725 & 0.76508 & 0.7687 & 0.76702 \\
\hline Sensitivity & 0.28903 & 0.31625 & 0.30147 & 0.3112 & 0.30547 \\
\hline Specificity & 0.92085 & 0.91756 & 0.9196 & 0.92118 & 0.92086 \\
\hline Precision & 0.54897 & 0.56114 & 0.55551 & 0.5682 & 0.56265 \\
\hline FPR & 0.079147 & 0.082436 & 0.080398 & 0.078823 & 0.079141 \\
\hline FNR & 0.71097 & 0.68375 & 0.69853 & 0.6888 & 0.69453 \\
\hline NPV & 0.92085 & 0.91756 & 0.9196 & 0.92118 & 0.92086 \\
\hline FDR & 0.45103 & 0.43886 & 0.44449 & 0.4318 & 0.43735 \\
\hline F1-score & 0.37869 & 0.40452 & 0.39084 & 0.40215 & 0.39597 \\
\hline MCC & 0.26882 & 0.29101 & 0.27955 & 0.29271 & 0.28614 \\
\hline
\end{tabular}

TABLE 5. ALGORITHMIC ANALYSIS ON TEST CASE 5

\begin{tabular}{|l|c|c|c|c|c|}
\hline Measures & $\tau=0.25$ & $\tau=0.40$ & $\tau=0.55$ & $\tau=0.70$ & $\tau=0.85$ \\
\hline Accuracy & 0.78037 & 0.78037 & 0.78002 & 0.78022 & 0.78201 \\
\hline Sensitivity & 0.36943 & 0.36919 & 0.36804 & 0.36817 & 0.36443 \\
\hline Specificity & 0.92478 & 0.92486 & 0.9248 & 0.92502 & 0.92875 \\
\hline Precision & 0.63315 & 0.63325 & 0.63235 & 0.63311 & 0.64254 \\
\hline FPR & 0.075222 & 0.075139 & 0.075196 & 0.074978 & 0.071246 \\
\hline FNR & 0.63057 & 0.63081 & 0.63196 & 0.63183 & 0.63557 \\
\hline NPV & 0.92478 & 0.92486 & 0.9248 & 0.92502 & 0.92875 \\
\hline FDR & 0.36685 & 0.36675 & 0.36765 & 0.36689 & 0.35746 \\
\hline F1-score & 0.4666 & 0.46644 & 0.46528 & 0.46559 & 0.46508 \\
\hline MCC & 0.35973 & 0.35966 & 0.35843 & 0.35898 & 0.36269 \\
\hline
\end{tabular}




\begin{tabular}{|l|c|c|c|c|c|}
\hline Measures & $\tau=0.25$ & $\tau=0.40$ & $\tau=0.55$ & $\tau=0.70$ & $\tau=0.85$ \\
\hline Accuracy & 0.80392 & 0.80298 & 0.80355 & 0.80265 & 0.80777 \\
\hline Sensitivity & 0.41031 & 0.41019 & 0.40872 & 0.40857 & 0.40864 \\
\hline Specificity & 0.92869 & 0.92749 & 0.92871 & 0.92756 & 0.93429 \\
\hline Precision & 0.64586 & 0.64198 & 0.64504 & 0.6413 & 0.66345 \\
\hline FPR & 0.071315 & 0.07251 & 0.071294 & 0.072439 & 0.065707 \\
\hline FNR & 0.58969 & 0.58981 & 0.59128 & 0.59143 & 0.59136 \\
\hline NPV & 0.92869 & 0.92749 & 0.92871 & 0.92756 & 0.93429 \\
\hline FDR & 0.35414 & 0.35802 & 0.35496 & 0.3587 & 0.33655 \\
\hline F1-score & 0.50182 & 0.50055 & 0.50038 & 0.49914 & 0.50576 \\
\hline MCC & 0.40268 & 0.40017 & 0.40123 & 0.39881 & 0.41256 \\
\hline
\end{tabular}

TABLE 7. ALGORITHMIC ANALYSIS ON TEST CASE 7

\begin{tabular}{|l|l|l|l|l|l|}
\hline Measures & $\tau=0.25$ & $\tau=0.40$ & $\tau=0.55$ & $\tau=0.70$ & $\tau=0.85$ \\
\hline Accuracy & 0.76798 & 0.76798 & 0.76762 & 0.76766 & 0.76996 \\
\hline Sensitivity & 0.38813 & 0.38793 & 0.38688 & 0.3865 & 0.38748 \\
\hline Specificity & 0.93759 & 0.93768 & 0.93762 & 0.93786 & 0.94074 \\
\hline Precision & 0.73522 & 0.7354 & 0.73469 & 0.73524 & 0.74486 \\
\hline FPR & 0.062411 & 0.062322 & 0.062384 & 0.062144 & 0.059262 \\
\hline FNR & 0.61187 & 0.61207 & 0.61312 & 0.6135 & 0.61252 \\
\hline NPV & 0.93759 & 0.93768 & 0.93762 & 0.93786 & 0.94074 \\
\hline FDR & 0.26478 & 0.2646 & 0.26531 & 0.26476 & 0.25514 \\
\hline F1-score & 0.50805 & 0.50792 & 0.50686 & 0.50666 & 0.50977 \\
\hline MCC & 0.40741 & 0.40739 & 0.40629 & 0.40639 & 0.41298 \\
\hline
\end{tabular}

\section{CONCLUSION}

This paper has presented an enhanced image segmentation technique using Active Contour, and Graph cut schemes. Here, the high-intensity and low-intensity pixels of the segmented images were clustered by FEM model, in which the maximization problem was solved by means of proposed AEW algorithm. By the exploitation of this adopted scheme, the segmentation accuracy was found to have improved in a better way. Moreover, algorithmic analysis was performed for the proposed system by varying the values of $\tau$ from $\tau$ $=0.25, \tau=0.40, \tau=0.55, \tau=0.70$ and $\tau=0.85$ in terms of relevant performance measures for 7 test cases. From the analysis, the accuracy of proposed model at $6^{\text {th }}$ test case for $\tau=0.25$ was $2.5 \%$ superior to $\tau=0.40,2.5 \%$ superior to $\tau=0.55,2.5 \%$ superior to $\tau=0.70$ and $2.5 \%$ superior to $\tau=0.85$. Thus the betterment of the presented scheme has been substantiated effectively.

\section{REFERENCES}

1. S. K. Choy, Kevin Yuen, Carisa Yu, "Fuzzy bit-plane-dependence image segmentation", Signal Processing, vol. 154, pp. 30-44, January 2019.

2. Li Guo, Long Chen, C. L. Philip Chen, Jin Zhou, "Integrating guided filter into fuzzy clustering for noisy image segmentation", Digital Signal Processing, vol. 83, pp. 235-248, December 2018.

3. PengGu, Won-MeanLee, Marilyn A.Roubidoux, JieYuan, XuedingWang and Paul L.Carson, "Automated 3D ultrasound image segmentation to aid breast cancer image interpretation", Ultrasonics", vol. 65, pp. 51-58, 2016.

4. Zexuan Ji, Yubo Huang, Yong Xia, Yuhui Zheng, “A robust segmentation", Neurocomputing, vol. 266, pp. 550-565, 29 November 2017.

5. Andrea Pennisi, Domenico D. Bloisi, Daniele Nardi, Anna Rita Giampetruzzi, Antonio Facchiano, "Skin lesion image segmentation using Delaunay Triangulation for melanoma modified Gaussian mixture model with rough set for image

detection", Computerized Medical Imaging and Graphics, vol. 52, pp. 89-103, September 2016.

6. Yiping Duan, Fang Liu, Licheng Jiao, Peng Zhao, Lu Zhang, “ SAR Image segmentation based on convolution-wavelet neura network and Markov random field", Pattern Recognition, vol. 64 pp. 255-267, April 2017.

7. M. Guijarro, I. Riomoros, G. Pajares, P. Zitinski, “ Discrete wavelets transform for improving greenness image segmentation in agricultural images", Computers and Electronics in Agriculture, vol.118, pp 396-407, October 2015

8. Jianning Chi, Mark Eramian, "Enhancing textural differences using wavelet-based texture characteristics morphological component analysis: A pre-processing method for improving image segmentation", Computer Vision and Image Understanding, vol.158, pp. 49-61, May 2017.

9. Zhang Yong, Yuan Jiazheng, Liu Hongzhe, Li Qing, "Grab-Cut image segmentation algorithm based on structure tensor", The Journal of China Universities of Posts and Telecommunications, vol. 24, no. 2, pp. 38-47, April 2017

10. Yafeng Li, Xiangchu Feng, "A multi-scale image segmentation method", Pattern Recognition, vol.52, pp. 332-345, April 2016.

11. Yongsheng Pan, Yong Xia, Tao Zhou, Michael Fulham, "Cell image segmentation using bacterial foraging optimization", Applied Soft Computing, vol. 58, pp.770-782, September 2017.

12. Weiwei Wang, Cuiling Wu, "Image segmentation by correlation adaptive weighted regression”, Neuro computing, 29 June 2017.

13. Iasonas Kokkinos; Petros Maragos, "Synergy between Object Recognition and Image Segmentation Using the Expectation-Maximization Algorithm", IEEE Journals \& Magazines, vol.31, no.08, pp.1486 - 1501, 2009.

14. Yousun Kang; Koichiro Yamaguchi; Takashi Naito; Yoshiki Ninomiya,"Multiband Image Segmentation and Object Recognition for Understanding Road Scenes", IEEE Journals \& Magazines, vol.12, no.04, pp.1423 - 1433, 2011

15. Fuzhuan Wu, Bingxin Wu,'Design and Realization of Fuzzy Control System for Textile Mill's Air-Conditioning Based on MSP430", IEEE Conference Publications, pp.1 - 4, 2010.

16. Gang Zheng; Yuanlu Li; Huinan Wang, "A New Multi-phase Level Set Framework for 3D Medical Image Segmentation Based on TPBG”, IEEE Conference Publications, pp.3394 - 3397, 2005 
17. Zhuowen Tu; Song-Chun Zhu, "Image segmentation by data-driven Markov chain Monte Carlo", IEEE Journals \& Magazines, vol.24, no.05, pp.657 - 673, 2002.

18. Kyumok Kim; Seung-Won Jung,“ Interactive Image Segmentation using Semi-transparent Wearable Glasses", IEEE Transactions on Multimedia, Vol. PP, no. 99, pp. 1 - 1, 2017.

19. Changjae Oh, Bumsub Ham, Kwanghoon Sohn, "Robust interactive image segmentation using structure-aware labelling", Expert Systems with Applications, vol. 79,pp. 90-100, 15 August 2017.

20. Hiren K. Mewada, Amit V. Patel, Keyur K. Mahant, "Concurrent design of active contour for image segmentation using Zynq ZC702", Computers \& Electrical Engineering, vol. 72, pp. 631-643, November 2018

21. Guowei Gao, Chenglin Wen, Huibin Wang, "Fast and robust image segmentation with active contours and Student's-t mixture model", Pattern Recognition, vol. 63, pp. 71-86, March 2017.

22. Seyedali Mirjalili, Andrew Lewis, "The Whale Optimization Algorithm", Advances in Engineering Software, vol. 95, pp. 51-67, May 2016. ultrasound volumes

23. M. Jogendra Kumar, and Dr. G.V.S. Raj Kumar, "Hybrid Image Segmentation Model based on Active Contour and Graph cut with Fuzzy Entropy Maximization", International Journal of Applied Engineering Research, vol.12, no.23, pp. 13623-13637, 2017.

24. G.V.S. Raj Kumar and M. Jogendra Kumar, "A Refined Image Segmentation Model Under Adaptiveness on Whale Optimization: AEW-AGFES", International Journal of Pure and Applied Mathematics, vol.119, no.16, pp.5119-5145, 2018. 